\title{
Over-the-Air Computation via Intelligent Reflecting Surfaces
}

\author{
Tao Jiang and Yuanming Shi \\ School of Information Science and Technology, ShanghaiTech University, Shanghai 201210, China \\ Email: \{jiangtao1, shiym\}@ shanghaitech.edu.cn
}

\begin{abstract}
Over-the-air computation (AirComp) becomes a promising approach for fast wireless data aggregation via exploiting the superposition property in a multiple access channel. To further overcome the unfavorable signal propagation conditions for AirComp, in this paper, we propose an intelligent reflecting surface (IRS) aided AirComp system to build controllable wireless environments, thereby boosting the received signal power significantly. This is achieved by smartly tuning the phase shifts for the incoming electromagnetic waves at IRS, resulting in reconfigurable signal propagations. Unfortunately, it turns out that the joint design problem for AirComp transceivers and IRS phase shifts becomes a highly intractable nonconvex biquadratic programming problem, for which a novel alternating difference-of-convex (DC) programming algorithm is developed. This is achieved by providing a novel DC function representation for the rank-one constraint in the low-rank matrix optimization problem via matrix lifting. Simulation results demonstrate the algorithmic advantages and admirable performance of the proposed approaches compared with the state-of-art solutions.
\end{abstract}

\section{INTRODUCTION}

In the near future, it is anticipated that massive number of Internet of things (IoT) devices and machines will be connected to wireless networks to automate the operations of our daily life, thereby providing intelligent services. To this end, one critical challenge is the need of ultra-fast wireless data aggregation, which pervades a wide range of applications in massive machine type communication [1] and on-device federated machine learning [2]. In particular, we need to collect and process data distributed among a huge number of devices rapidly by wireless communication techniques. However, with enormous number of devices, conventional interference-avoiding channel access schemes become infeasible since they normally result in low spectrum utilization efficiency and excessive network latency [3]. To overcome this challenge, a promising concurrent transmissions solution named over-the-air computation (AirComp) was proposed via exploiting the superposition property in a multiple access channel [4]-[6].

There are extensive research works on investigating the AirComp systems from the point of view of information theory [4], signal processing [5], [7], and transceiver beamforming design [3], [6], [8]. In particular, a uniform-forcing transceiver design was developed in [6] via the successive convex approximation method to compensate the non-uniform fading of different sensors. A novel transmitter design leveraging zeroforcing beamforming has recently been proposed in [8] to compensate the non-uniform fading among different multiple antennas at IoT devices. In [3], a multiple-input-multiple output (MIMO) AirComp scheme was further investigated to enable high-mobility multi-modal sensing. It showed that more antennas at access point (AP) is able to reduce the performance degradation in terms of mean-squared-error (MSE). However, all these approaches are unable to control of the wireless environments, where in some scenarios the harsh propagation environments may result in significant deterioration of the system performance [9]. For instance, high frequency (e.g., millimeter wave or terahertz) signals, which are expected to play a key role in future communication systems, however, may be blocked even by small objects [9].

To overcome unfavorable signal propagation conditions for AirComp, in this paper, we propose to boost the performance of AirComp by developing large intelligent reflecting surfaces (IRS), which is envisioned to achieve high spectrum and energy efficiency by controlling the communication environments [10], [11]. An IRS normally does not require any dedicated energy source and can be integrated easily in the surrounding walls of the transmitters [12], [13]. Specifically, an IRS is generally composed of many small passive elements, each of which is able to reflect a phase-shifted version of the incident signal [12]-[15]. By intelligently tuning the phase shifts, we are able to constructively combine reflected signals with the non-reflected ones to boost the received signal power drastically, thereby improving the achievable performance of AirComp.

Although there is a growing body of recent works on transmit beamforming and IRS phase shifts design [12], [13], the transceiver design for AirComp raises unique challenges due to the coupled design of the optimal phase shifts of a large IRS. In this paper, we propose to jointly optimize the transceiver and the phase shifts to minimize the MSE for AirComp. However, it turns out that the joint design problem for AirComp transceivers and IRS phase shifts becomes a highly intractable nonconvex bi-quadratic programming problem. In order to address the coupled issue, we propose to optimize the phase shifts and the decoding vector at the AP alternatively. It turns out that the decoding vector design problem for AirComp [3] and the phase shifts matrix design problem for IRS [13] are both nonconvex quadratically constrained quadratic programming (QCQP) problems.

A popular way to convexify the nonconvex QCQP problem is to reformulate it as a rank-one constrained matrix optimization problem via matrix lifting, followed by the semidefinite 
relaxation (SDR) technique to drop the nonconvex rank-one constraint [16]. However, it was observed that the performance of SDR approach degenerates in the scenarios with large number of antennas due to its low probability of returning rank-one solutions [2], [6], [17]. To address the limitations of the popular SDR technique, in this paper, we develop a general framework to solve the rank-one constrained matrix optimization problem via difference-of-convex (DC) programming. This is achieved by providing a novel DC function representation for the rank-one constraint, followed by a majorization-minimization algorithm to solve the resulting DC problem. Furthermore, simulation results demonstrate that the proposed approach outperforms the SDR method significantly, and large IRS is able to dramatically enhance the AirComp performance.

Notations: $\|\cdot\|,(\cdot)^{\top},(\cdot)^{\mathrm{H}}$ and $\operatorname{Tr}(\cdot)$ denote Euclidian norm, transpose, conjugate transpose and trace operators, respectively. $\boldsymbol{Q} \sim \mathcal{C N}\left(\mu, \sigma^{2} \boldsymbol{I}\right)$ stands for each element in $\boldsymbol{Q}$ following i.i.d. normal distribution with mean $\mu$ and variance $\sigma^{2}$.

\section{System Model AND PROBlem FORMUlation}

\section{A. System Model}

We consider a multi-user MISO communication system consisting of $K$ single-antenna users and an AP with $N$ antennas. In the scenario of over-the-air computation, the AP aims to compute a target function of the aggregated data from all users [3], [6], as shown in Fig. 1. Specifically, let $x_{k} \in \mathbb{C}$ denote data generated at user $k$ and $\psi_{k}(\cdot): \mathbb{C} \rightarrow \mathbb{C}$ denote the pre-processing function of user $k$, the target function computed at AP can be written in the form as

$$
f=\phi\left(\sum_{k=1}^{K} \psi_{k}\left(x_{k}\right)\right)
$$

where $\phi(\cdot)$ is the post-processing function of AP. Denote $s_{k}:=$ $\psi\left(x_{k}\right)$ as the transmitted symbols at user $k$. The transmitted symbols are assumed to be normalized to have unit variance, i.e., $\mathbb{E}\left(s_{k} s_{k}^{\mathrm{H}}\right)=1$, and $\mathbb{E}\left(s_{k} s_{j}^{\mathrm{H}}\right)=0, \forall k \neq j$. To compute the target function $f$, AP needs to obtain the target-function variable defined as

$$
s:=\sum_{k=1}^{K} s_{k}
$$

In this paper, we aim to recover this target-function variable by exploiting the superposition property of a wireless multipleaccess channel.

To enhance the performance for over-the-air computation by controlling the signal propagation environment, we shall propose to deploy an intelligent reflecting surface (IRS) on a surrounding wall, thereby dynamically adjusting the phase shift of each reflecting elements according to the channel state information (CSI). In particular, the IRS controller can switch between two operational modes, i.e., the receiving mode for sensing the environment (e.g., CSI estimation) and the reflecting mode for scattering the incident signals from the users [13], [14]. The IRS has $M$ elements, each of which rescatters the received incident signals with a phase shift and a

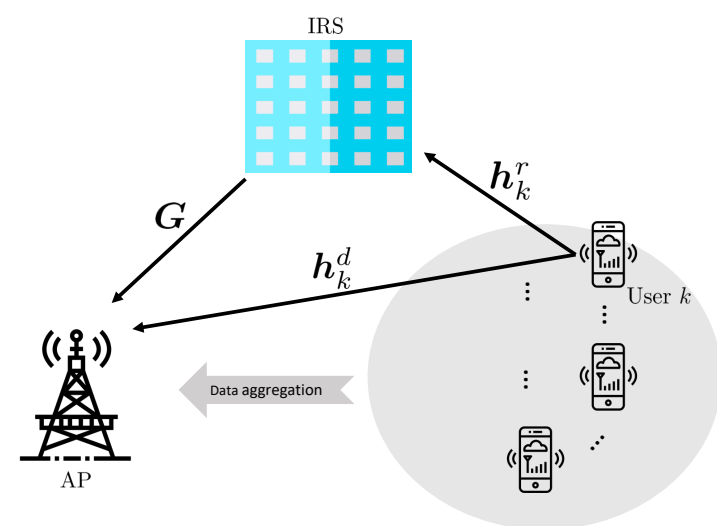

Fig. 1. Over-the-air computation with intelligent reflecting surface.

magnitude loss. Specifically, let $\boldsymbol{\Theta}=\operatorname{diag}\left(\beta e^{j \theta_{1}}, \cdots, \beta e^{j \theta_{M}}\right)$ represent the diagonal phase shifts matrix of the IRS with $\theta_{m} \in[0,2 \pi]$ and $\beta \in[0,1]$ as the amplitude reflection coefficient on the incident signals. In this paper, we assume $\beta=1$ without loss of generality. Furthermore, it is well known that the power of signals reflected by twice or more times can be ignored due to significant propagation loss [13]. Therefore, the equivalent uplink channel between users and AP consists of three components, i.e., AP-user link, IRS-user link, and IRS-AP link as show in Fig. 1. Additionally, we assume all the involved channels are constant during a block of transmission.

Let $\boldsymbol{h}_{k}^{d} \in \mathbb{C}^{N}, \boldsymbol{h}_{k}^{r} \in \mathbb{C}^{M}$, and $\boldsymbol{G} \in \mathbb{C}^{N \times M}$ be the equivalent channels from user $k$ to the AP, from user $k$ to the IRS, and from IRS to the AP, respectively. The received signal at AP is thus given by

$$
\boldsymbol{y}=\sum_{k=1}^{K}\left(\boldsymbol{G} \Theta \boldsymbol{h}_{k}^{r}+\boldsymbol{h}_{k}^{d}\right) w_{k} s_{k}+\boldsymbol{n}
$$

where $w_{k} \in \mathbb{C}$ is the transmitter scalar and $\boldsymbol{n} \in \mathbb{C}^{N} \sim$ $\mathcal{C N}\left(0, \sigma^{2} \boldsymbol{I}\right)$ is the additive white Gaussian noise. Transmission power at each user can not exceed a given positive value $P_{0}$, namely,

$$
\left|w_{k}\right|^{2} \leq P_{0}, \forall k \text {. }
$$

Given a decoding vector $\boldsymbol{m} \in \mathbb{C}^{N}$ at the AP, the estimated target function variable is given by

$\hat{s}=\frac{1}{\sqrt{\eta}} \boldsymbol{m}^{\mathrm{H}} \boldsymbol{y}=\frac{1}{\sqrt{\eta}} \boldsymbol{m}^{\mathrm{H}} \sum_{k=1}^{K}\left(\boldsymbol{G} \boldsymbol{\Theta} \boldsymbol{h}_{k}^{r}+\boldsymbol{h}_{k}^{d}\right) w_{k} s_{k}+\frac{1}{\sqrt{\eta}} \boldsymbol{m}^{\mathrm{H}} \boldsymbol{n}$,

where $\eta$ is a normalizing factor.

\section{B. Problem Formulation}

In this paper, we aim to minimize the distortion after decoding, which is measured by the MSE defined as follows

$$
\begin{aligned}
\mathrm{MSE} & :=\mathbb{E}\left(|\hat{s}-s|^{2}\right) \\
& =\sum_{k=1}^{K}\left|\frac{1}{\sqrt{\eta}} \boldsymbol{m}^{H} \boldsymbol{h}_{k}^{e} w_{k}-1\right|^{2}+\frac{\sigma^{2}\|\boldsymbol{m}\|^{2}}{\eta},
\end{aligned}
$$


where $\boldsymbol{h}_{k}^{e}=\boldsymbol{G} \boldsymbol{\Theta} \boldsymbol{h}_{k}^{r}+\boldsymbol{h}_{k}^{d}$ denotes the combined AP-user channel vector. To minimize the MSE, we need to seek the optimal transceivers $w_{k}$ 's, $\boldsymbol{m}$, and the phase shifts matrix $\boldsymbol{\Theta}$.

Given the decoding vector $\boldsymbol{m}$ and the phase shifts matrix $\boldsymbol{\Theta}$, the optimal transmitter scalars can be designed as [2], [6]

$$
w_{k}=\sqrt{\eta} \frac{\left(\boldsymbol{m}^{\mathrm{H}} \boldsymbol{h}_{k}^{e}\right)^{\mathrm{H}}}{\left\|\boldsymbol{m}^{\mathrm{H}} \boldsymbol{h}_{k}^{e}\right\|^{2}}, \forall k,
$$

where $\eta$ is calculated by

$$
\eta=P_{0} \min _{k}\left\|\boldsymbol{m}^{\mathrm{H}} \boldsymbol{h}_{k}^{e}\right\|^{2},
$$

to satisfy the power constraint (4) on each transmitter scalar.

Given the optimal transmitter scalars $w_{k}$ 's and the normalizing factor $\eta$, the MSE can be further rewritten as

$$
\mathrm{MSE}=\frac{\sigma^{2}\|\boldsymbol{m}\|^{2}}{P_{0} \min _{k}\left\|\boldsymbol{m}^{\mathrm{H}}\left(\boldsymbol{G} \boldsymbol{\Theta} \boldsymbol{h}_{k}^{r}+\boldsymbol{h}_{k}^{d}\right)\right\|^{2}} .
$$

We thus propose to jointly optimize the phase shifts matrix $\Theta$ and the decoding vector $\boldsymbol{m}$ to minimize the MSE as follows:

$$
\begin{aligned}
& \underset{\boldsymbol{m}, \boldsymbol{\Theta}}{\operatorname{minimize}}\left(\max _{k} \frac{\|\boldsymbol{m}\|^{2}}{\left\|\boldsymbol{m}^{\mathrm{H}}\left(\boldsymbol{G} \boldsymbol{\Theta} \boldsymbol{h}_{k}^{r}+\boldsymbol{h}_{k}^{d}\right)\right\|^{2}}\right) \\
& \text { subject to } \quad 0 \leq \theta_{n} \leq 2 \pi, \forall n=1, \cdots, N .
\end{aligned}
$$

We further equivalently reformulate problem (10) as the following bi-quadratic programming problem:

$$
\begin{aligned}
\mathscr{P}: \underset{\boldsymbol{m}, \boldsymbol{\Theta}}{\operatorname{minimize}} & \|\boldsymbol{m}\|^{2} \\
\text { subject to } & \left\|\boldsymbol{m}^{\mathrm{H}}\left(\boldsymbol{G} \boldsymbol{\Theta} \boldsymbol{h}_{k}^{r}+\boldsymbol{h}_{k}^{d}\right)\right\|^{2} \geq 1, \forall k, \\
& 0 \leq \theta_{n} \leq 2 \pi, \forall n=1, \cdots, N .
\end{aligned}
$$

However, problem $\mathscr{P}$ turns out to be highly intractable due to the nonconvex quadratic constraints with respect to $\boldsymbol{m}$ and $\Theta$. In Section III, we shall leverage the alternating minimization approach to solve this problem. A novel alternating DC programming algorithm is further developed in Section IV.

\section{Alternating Minimization}

In this section, we propose to solve problem $\mathscr{P}$ by the alternating minimization approach. Specifically, the decoding vector $\boldsymbol{m}$ at AP and the phase shifts matrix $\Theta$ at the IRS are optimized in an alternative manner until the algorithm converges.

\section{A. Alternating Minimization}

For given phase shifts matrix $\Theta$, problem $\mathscr{P}$ becomes the following nonconvex QCQP problem

$$
\begin{array}{ll}
\underset{\boldsymbol{m}}{\operatorname{minimize}} & \|\boldsymbol{m}\|^{2} \\
\text { subject to } & \left\|\boldsymbol{m}^{\mathrm{H}} \boldsymbol{h}_{k}^{e}\right\|^{2} \geq 1, \forall k .
\end{array}
$$

On the other hand, for a given decoding vector $\boldsymbol{m}$, problem $\mathscr{P}$ is reduced to a feasibility detection problem. Specifically, let $\boldsymbol{m}^{\mathrm{H}} \boldsymbol{h}_{k}^{d}=c_{k}$ and $v_{m}=e^{j \theta_{m}}, m=1, \cdots, M$, we have
$\boldsymbol{m}^{\mathrm{H}} \boldsymbol{G} \boldsymbol{\Theta} \boldsymbol{h}_{k}^{r}=\boldsymbol{a}_{k}^{\mathrm{H}} \boldsymbol{v}$, where $\boldsymbol{v}=\left[e^{j \theta_{1}}, \cdots, e^{j \theta_{M}}\right]^{\boldsymbol{\top}}$ and $\boldsymbol{a}_{k}^{\mathrm{H}}=$ $\boldsymbol{m}^{\mathrm{H}} \boldsymbol{G} \operatorname{diag}\left(\boldsymbol{h}_{k}^{r}\right)$. Therefore, problem $\mathscr{P}$ can be written as

$$
\begin{aligned}
\text { find } & \boldsymbol{v} \\
\text { subject to } & \left|\boldsymbol{a}_{k}^{\mathrm{H}} \boldsymbol{v}+c_{k}\right|^{2} \geq 1, \forall k, \\
& \left|v_{n}\right|^{2}=1, \forall v=1, \cdots, N .
\end{aligned}
$$

Although problem (13) is nonconvex and inhomogeneous, it can be reformulated as a homogeneous nonconvex QCQP problem [13]. Specifically, by introducing an auxiliary variable $t$, we can equivalently rewrite problem (13) as

$$
\begin{aligned}
\text { find } & \boldsymbol{v} \\
\text { subject to } & \tilde{\boldsymbol{v}}^{\mathrm{H}} \boldsymbol{R}_{k} \tilde{\boldsymbol{v}}+c_{k}^{2} \geq 1, \forall k, \\
& \left|v_{n}\right|^{2}=1, \forall v=1, \cdots, N,
\end{aligned}
$$

where

$$
\boldsymbol{R}_{k}=\left[\begin{array}{cc}
\boldsymbol{a}_{k} \boldsymbol{a}_{k}^{\mathrm{H}}, & \boldsymbol{a}_{k} c_{k} \\
c_{k}^{\mathrm{H}} \boldsymbol{a}_{k}^{\mathrm{H}}, & 0
\end{array}\right], \quad \tilde{\boldsymbol{v}}=\left[\begin{array}{l}
\boldsymbol{v} \\
t
\end{array}\right] .
$$

Obviously, if $\tilde{\boldsymbol{v}}^{*}=\left[\boldsymbol{v}^{0}, t^{0}\right]^{\top}$ is a feasible solution to problem (14), then we can obtain a feasible solution to problem (13) as $\boldsymbol{v}^{*}=\boldsymbol{v}^{0} / t^{0}$. The phase shifts matrix $\boldsymbol{\Theta}^{*}$ can be recovered from $v^{*}$ trivially. Note that problem (12) is always feasible, while the feasibility of problem (14) may not be guaranteed. We thus terminate the alternating algorithm either problem (14) becomes infeasible during the iterative procedure or the difference between the MSE of consecutive iterations is less than a predefined threshold.

To summarize, we propose to solve the nonconvex biquadratic problem $\mathscr{P}$ by seeking the optimal solution to problem (12) and problem (14) in an alternative manner. Although both problem (12) and problem (14) are still non convex, we shall reveal the algorithmic advantages in the following sections.

\section{B. Matrix Lifting}

To address the nonconvexity issue of problem (12) and problem (14), a natural way is to reformulate them as semidefinite programming (SDP) problems by the matrix lifting technique [16]. Specifically, by defining $\boldsymbol{M}=\boldsymbol{m} \boldsymbol{m}^{\mathrm{H}}$, which lifts the vector $\boldsymbol{m}$ into a positive semidefinite (PSD) matrix with $\operatorname{rank}(\boldsymbol{M})=1$, problem (12) can be equivalently reformulated as the following low-rank matrix optimization problem

$$
\begin{aligned}
& \mathscr{P}_{1}: \underset{\boldsymbol{M}}{\operatorname{minimize}} \operatorname{Tr}(\boldsymbol{M}) \\
& \text { subject to } \operatorname{Tr}\left(\boldsymbol{M} \boldsymbol{H}_{k}\right) \geq 1, \forall k \text {, } \\
& \boldsymbol{M} \succeq 0, \operatorname{rank}(\boldsymbol{M})=1 \text {, }
\end{aligned}
$$

where $\boldsymbol{H}_{k}=\boldsymbol{h}_{k}^{e}\left(\boldsymbol{h}_{k}^{e}\right)^{\mathrm{H}}$.

Similarly, we also adopt the matrix lifting technique to reformulate the nonconvex quadratic constraints in problem (14). Specifically, let $\boldsymbol{V}=\tilde{\boldsymbol{v}} \tilde{\boldsymbol{v}}^{\mathrm{H}}$ and note that $\tilde{\boldsymbol{v}}^{\mathrm{H}} \boldsymbol{R}_{k} \tilde{\boldsymbol{v}}=$ 
$\operatorname{Tr}\left(\boldsymbol{R}_{k} \tilde{\boldsymbol{v}} \tilde{\boldsymbol{v}}^{\mathrm{H}}\right)$. Problem (14) can be equivalently written as the following low-rank matrix optimization problem

$$
\begin{array}{cl}
\mathscr{P}_{2}: \text { find } & \boldsymbol{V} \\
\text { subject to } & \operatorname{Tr}\left(\boldsymbol{R}_{k} \boldsymbol{V}\right)+c_{k}^{2} \geq 1, \forall k, \\
& \boldsymbol{V}_{n, n}=1, \forall n=1, \cdots, N, \\
& \boldsymbol{V} \succeq 0, \quad \operatorname{rank}(\boldsymbol{V})=1 .
\end{array}
$$

\section{Problem Analysis}

To further address the nonconvexity in problem $\mathscr{P}_{1}$ and problem $\mathscr{P}_{2}$, one popular way is to simply drop the nonconvex rank-one constraints via the SDR technique [16]. The resulting SDP problems can be solved efficiently by existing convex optimization solvers such as CVX [18]. If the optimal solution to the SDP problem is rank-one, the optimal solution to the original problem can be recovered by rank one decomposition. On the other hand, if the optimal solution of the SDP problem fails to be rank-one, additional steps such as Gaussian randomization [16] need to be applied to extract a suboptimal solution for the original problem. However, it was observed that for the high-dimensional optimization problems (e.g., the number of antennas $N$ increases), the probability of returning a rank-one solution for the SDR approach becomes low, which yields significant performance deterioration [6], [17]. To overcome the limitations of the SDR methods, we instead propose a novel DC framework in the following section to solve problem $\mathscr{P}_{1}$ and problem $\mathscr{P}_{2}$.

\section{Alternating DC Algorithm}

In this section, we will introduce a novel DC representation for the rank function, following by leveraging the majorization-minimization technique to iteratively solve the resulting DC problems.

\section{A. DC Framework for Rank-One Constraint Problems}

For ease of presentation, we first consider the DC algorithm for general low-rank matrix optimization problems with a rank-one constraint as follows,

$$
\begin{array}{cl}
\underset{\boldsymbol{X} \in \mathcal{C}}{\operatorname{minimize}} & \operatorname{Tr}\left(\boldsymbol{A}_{0} \boldsymbol{X}\right) \\
\text { subject to } & \operatorname{Tr}\left(\boldsymbol{A}_{k} \boldsymbol{X}\right) \geq d_{k}, \forall k, \\
& \boldsymbol{X} \succeq 0, \operatorname{rank}(\boldsymbol{X})=1,
\end{array}
$$

where the constraint set $\mathcal{C}$ is convex. A key observation on the rank-one constraint is that it can be equivalently written as a DC function constraint, which is formally stated in the following proposition [2].

Proposition 1. For positive semidefinite (PSD) matrix $\boldsymbol{X} \in$ $\mathbb{C}^{N \times N}$ and $\operatorname{Tr}(\boldsymbol{X}) \geq 1$, we have

$$
\operatorname{rank}(\boldsymbol{X})=1 \Longleftrightarrow \operatorname{Tr}(\boldsymbol{X})-\|\boldsymbol{X}\|_{2}=0,
$$

where trace norm $\operatorname{Tr}(\boldsymbol{X})=\sum_{i=1}^{N} \sigma_{i}(\boldsymbol{X})$ and spectral norm $\|\boldsymbol{X}\|_{2}=\sigma_{1}(\boldsymbol{X})$ with $\sigma_{i}(\boldsymbol{X})$ denoting the $i$-th largest singular value of matrix $\boldsymbol{X}$.

In order to enhance a low rank solution for problem (18), instead of removing the nonconvex rank-one constraint via the
SDR method, we propose to add the DC function in (19) into the objective function as a penalty component, yielding

$$
\begin{array}{cl}
\underset{\boldsymbol{X} \in \mathcal{C}}{\operatorname{minimize}} & \operatorname{Tr}\left(\boldsymbol{A}_{0} \boldsymbol{X}\right)+\rho \cdot\left(\operatorname{Tr}(\boldsymbol{X})-\|\boldsymbol{X}\|_{2}\right) \\
\text { subject to } & \operatorname{Tr}\left(\boldsymbol{A}_{k} \boldsymbol{X}\right) \geq d_{k}, \forall k, \\
& \boldsymbol{X} \succeq 0,
\end{array}
$$

where $\rho>0$ is the penalty parameter. Note that we are able to obtain an exact rank-one solution $\boldsymbol{X}^{*}$ when the nonnegative component $\left(\operatorname{Tr}\left(\boldsymbol{X}^{*}\right)-\left\|\boldsymbol{X}^{*}\right\|_{2}\right)$ in the objective function is enforced to be zero.

\section{B. DC Algorithm}

Although problem (20) is still nonconvex, it can be solved in an iterative manner by leveraging majorization-minimization techniques, yielding a DC algorithm [19]. The main idea is to transform problem (20) into a series of simple subproblems by linearizing the concave term $-\rho\|\boldsymbol{X}\|_{2}$ in the objective function. Specifically, we need to solve the subproblem at iteration $t$ which is given by

$$
\begin{array}{cl}
\underset{\boldsymbol{X} \in \mathcal{C}}{\operatorname{minimize}} & \operatorname{Tr}\left(\boldsymbol{A}_{0} \boldsymbol{X}\right)+\rho \cdot\left\langle\boldsymbol{X}, \boldsymbol{I}-\partial\left\|\boldsymbol{X}^{t-1}\right\|_{2}\right\rangle \\
\text { subject to } & \operatorname{Tr}\left(\boldsymbol{A}_{k} \boldsymbol{X}\right) \geq d_{k}, \forall k, \\
& \boldsymbol{X} \succeq 0,
\end{array}
$$

where $\boldsymbol{X}^{t-1}$ is the optimal solution of the subproblem at iteration $t-1$. It is clear that the subproblem (21) is convex and can be solved efficiently by existing solvers such as CVX [18]. In addition, the subgradient $\partial\|\boldsymbol{X}\|_{2}$ can be computed efficiently by the following proposition [2].

Proposition 2. For given PSD matrix $\boldsymbol{X} \in \mathbb{C}^{N \times N}$, the subgradient $\partial\|\boldsymbol{X}\|_{2}$ can be computed as $\boldsymbol{v}_{1} \boldsymbol{v}_{1}^{\mathrm{H}}$, where $\boldsymbol{v}_{1} \in \mathbb{C}^{N}$ $i$ s the leading eigenvector of matrix $\boldsymbol{X}$.

The presented DC algorithm converges to critical points of problem (20) from arbitrary initial points [19]. We thus summarize the presented DC algorithm in Algorithm 1.

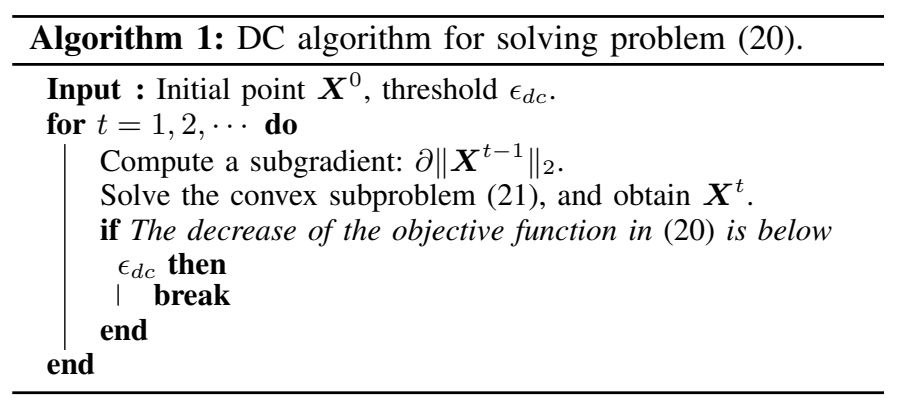

\section{Proposed Alternating DC Approach}

In this subsection, we apply the proposed DC framework to problem $\mathscr{P}_{1}$ and problem $\mathscr{P}_{2}$. Specifically, to find a rank-one solution to problem $\mathscr{P}_{1}$, we propose to solve the following DC programming problem

$$
\begin{array}{cl}
\underset{\boldsymbol{M}}{\operatorname{minimize}} & \operatorname{Tr}(\boldsymbol{M})+\rho\left(\operatorname{Tr}(\boldsymbol{M})-\|\boldsymbol{M}\|_{2}\right) \\
\text { subject to } & \operatorname{Tr}\left(\boldsymbol{M} \boldsymbol{H}_{k}\right) \geq 1, \forall k, \\
& \boldsymbol{M} \succeq 0,
\end{array}
$$


where $\rho>0$ is the penalty parameter. When the penalty component is enforced to be zero, problem (22) shall induce a rank-one solution $M^{\star}$, we can thus recover the solution $\boldsymbol{m}$ to problem (12) through Cholesky decomposition $\boldsymbol{M}^{\star}=\boldsymbol{m} \boldsymbol{m}^{\mathrm{H}}$.

To detect feasibility for problem $\mathscr{P}_{2}$, we propose to minimize the difference between trace norm and spectral norm as follows,

$$
\begin{array}{cl}
\underset{\boldsymbol{V}}{\operatorname{minimize}} & \operatorname{Tr}(\boldsymbol{V})-\|\boldsymbol{V}\|_{2} \\
\text { subject to } & \operatorname{Tr}\left(\boldsymbol{R}_{k} \boldsymbol{V}\right)+c_{k}^{2} \geq 1, \forall k, \\
& \boldsymbol{V}_{n, n}=1, \forall n=1, \cdots, N, \\
& \boldsymbol{V} \succeq 0 .
\end{array}
$$

When the objective value of problem (23) becomes zero, we shall find an exact rank-one optimal solution $\boldsymbol{V}^{\star}$. By Cholesky decomposition $\boldsymbol{V}^{\star}=\tilde{\boldsymbol{v}} \tilde{\boldsymbol{v}}^{\mathrm{H}}$, we can obtain a feasible solution $\tilde{\boldsymbol{v}}$ to problem (14). If the objective value fails to be zero, we claim that problem $\mathscr{P}_{2}$ (i.e., problem (14)) is infeasible.

In summary, the proposed alternating DC algorithm for solving problem $\mathscr{P}$ can be presented in Algorithm 2.

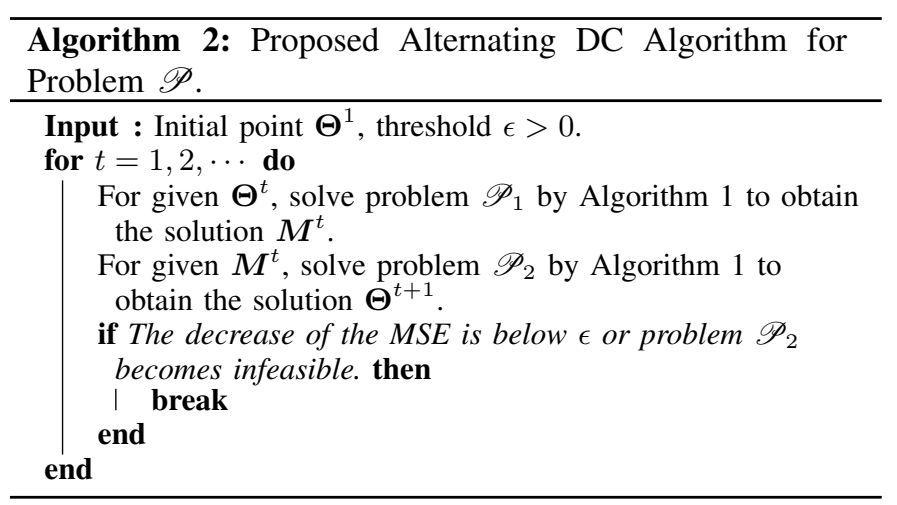

\section{Simulations}

In this section, we conduct numerical experiments to evaluate the performance of the proposed alternating DC algorithm for solving problem $\mathscr{P}$ and the effectiveness of IRS for the over-the-air computation systems.

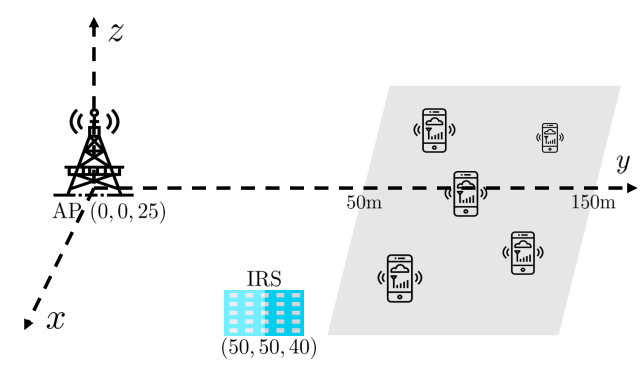

Fig. 2. Layout of AP, IRS and users.

\section{A. Simulation Settings}

We consider a three-dimensional (3D) coordinate system with a uniform linear array of antennas at the AP and a uniform rectangular array of passive reflecting elements at the IRS, respectively. The AP and the IRS are respectively located at $(0,0,25)$ meters and $(50,50,40)$ meters, while the users are are uniformly located at region $[-50,50] \times[50,150]$ meters as illustrated in Fig. 2. We consider the following pass loss model

$$
L(d)=T_{0}\left(\frac{d}{d_{0}}\right)^{-\alpha},
$$

where $T_{0}$ is the path loss at the reference distance $d_{0}=1 \mathrm{~m}, \mathrm{~d}$ is the link distance and $\alpha$ is the path loss exponent. In simulations, we set $T_{0}=30 \mathrm{~dB}$, and the path loss exponent $\alpha$ for APuser link, AP-IRS link and IRS-user link are respectively set to be $3.5,2.2,2.8$. In addition, we assume Rayleigh fading for all the considered channels. Specifically, the channel coefficients are given by $\boldsymbol{h}_{k}^{d}=\sqrt{L\left(d_{k}^{d}\right)} \boldsymbol{\gamma}^{d}$ and $\boldsymbol{h}_{k}^{d}=\sqrt{L\left(d_{k}^{r}\right)} \gamma^{r}$, where $\gamma^{d} \sim \mathcal{C N}(0, \boldsymbol{I}), \gamma^{r} \sim \mathcal{C N}(0, \boldsymbol{I})$, Here, $d_{k}^{d}$ and $d_{k}^{r}$ are the distance between user $k$ and AP, the distance between user $k$ and IRS, respectively. The channel matrix $\boldsymbol{G}=\sqrt{L(d)} \boldsymbol{\Gamma}$, where $\boldsymbol{\Gamma} \sim \mathcal{C N}(0, \boldsymbol{I})$ and $d$ is the distance between AP and IRS. The average transmit signal-to-noise-ratio (SNR) $P_{0} / \sigma^{2}$ is set to be $30 \mathrm{~dB}$. The other parameters are set as follows: $\rho=5, \epsilon=10^{-3}, \epsilon_{d c}=10^{-8}$.

We compare our proposed alternating DC algorithm with the alternating SDR method for solving problem $\mathscr{P}$, i.e., the SDR method is adopted to solve both problem $\mathscr{P}_{1}$ [16] and problem $\mathscr{P}_{2}$ [13]. For the SDR method, we remove the rankone constraint in problem $\mathscr{P}_{1}$ and problem $\mathscr{P}_{2}$ and solve them alternatively via CVX [18], and we stop it when the difference between the MSE of consecutive iterations is below $\epsilon$ or the SDR approach fails to return a feasible solution to problem (14). Since the SDR method does not generally return a rank-one solution, we apply Gaussian randomization techniques [16] when we fail to obtain a rank-one solution. We also illustrate the results given by random phase shift method as the baseline. That is, to solve problem $\mathscr{P}$, we choose a fixed random phase shifts matrix $\Theta$ and minimize the MSE by solving problem $\mathscr{P}_{1}$ via proposed DC Algorithm 1 .

\section{B. Simulation Results}

We show the convergence behavior of the proposed alternating DC algorithm and alternating SDR method in Fig. 3(a) under the setting: $K=16, M=30, N=20$. It demonstrates that the alternating SDR method stops at the third iteration since it fails to find a feasible solution to problem (14) even with Gaussian randomization techniques. However, the proposed alternating DC algorithm is able to induce exact rank-one optimal solutions, thereby accurately detecting the feasibility of problem (14). This yields good performance with a small MSE overall.

We compare in Fig. 3(b) the MSE versus different numbers of the AP antennas $N$. The number of elements at IRS is fixed to $M=15$ and the number of users is $K=8$. Each point in Fig. 3(b) is averaged over 100 channel realizations. As can be seen from Fig. 3(b), the MSE decreases significantly as $N$ increases, which indicates more antennas at AP will bring better performance for AirComp. Furthermore, the proposed 


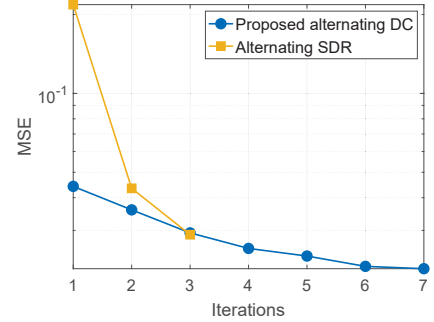

(a) Convergence results.

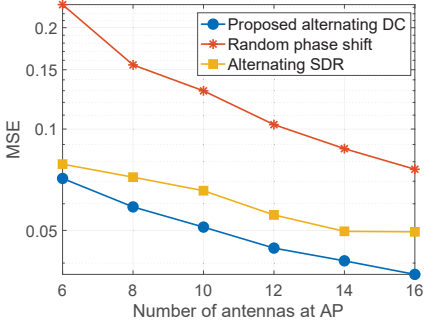

(b) MSE vs. number of AP antennas.

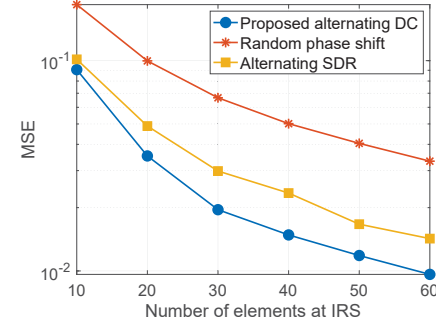

(c) MSE vs. number of IRS elements.

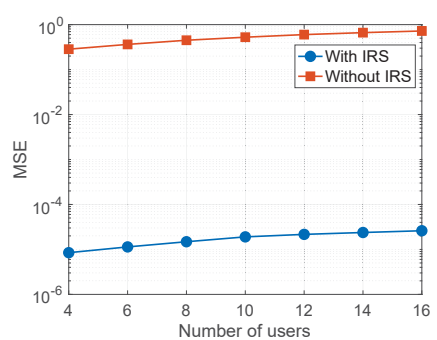

(d) MSE vs. number of users

Fig. 3. Performance of different algorithms for solving problem $\mathscr{P}$.

alternating DC approach significantly outperforms alternating SDR methods and the baseline.

We further compare in Fig. 3(c) the MSE versus different number of IRS elements $M$ with fixed $N=10, K=8$. Each point in Fig. 3(c) is averaged over 100 channel realizations. From Fig. 3(c), it illustrates that as $M$ increases, the MSE decreases significantly, which indicates that IRS with larger number of elements is able to achieve smaller MSE. In addition, the proposed alternating DC approach outperforms alternating SDR methods significantly.

Finally, we compare the performance between AirComp with IRS and the one without IRS. We fix the number of the AP antennas $N=8$. For the case without IRS, we set the phase shifts matrix $\Theta=\mathbf{0}$ in problem $\mathscr{P}$, and minimize the MSE by solving problem (22) using the proposed DC Algorithm 1. For the case with IRS, we fix the the number of the IRS elements $M=15$. We illustrate the results of the MSE versus the number of users in Fig. 3(d), and each point is averaged over 100 channel realizations. It shows that the MSE performance of the case without IRS is bad, which suggests that deploying IRS in AirComp system can significantly enhance its performance.

\section{CONCLUSION}

In this paper, we proposed to leverage the large intelligent surfaces to boost the performance for over-the-air computation, thereby achieving ultra-fast data aggregation. To find the optimal transceiver and phase shifts design, we proposed an alternating minimization based approach, which results in solving the nonconvex QCQP problems alternatively. To overcome the nonconvexity issue, we further reformulated the QCQP problems as a rank-one constrained matrix optimization problem via matrix lifting, followed by a novel DC framework to address the nonconvex rank-one constraints. Simulation results demonstrated the admirable performance of the proposed approaches compared with the state-of-the-art algorithms.

\section{REFERENCES}

[1] J. Guo, S. Durrani, X. Zhou, and H. Yanikomeroglu, "Massive machine type communication with data aggregation and resource scheduling," IEEE Trans. Commun., vol. 65, no. 9, pp. 4012-4026, Sep. 2017.

[2] K. Yang, T. Jiang, Y. Shi, and Z. Ding, "Federated learning based on over-the-air computation," in Proc. IEEE Int. Conf. Commun. (ICC), Shanghai, China, May 2019.
[3] G. Zhu and K. Huang, "MIMO over-the-air computation for highmobility multi-modal sensing," IEEE Internet Things J., to appear 2018.

[4] B. Nazer and M. Gastpar, "Computation over multiple-access channels," IEEE Trans. Inf. Theory, vol. 53, no. 10, pp. 3498-3516, Oct. 2007.

[5] M. Goldenbaum, H. Boche, and S. Stańczak, "Harnessing interference for analog function computation in wireless sensor networks," IEEE Trans. Signal Process, vol. 61, no. 20, pp. 4893-4906, Oct. 2013.

[6] L. Chen, X. Qin, and G. Wei, "A uniform-forcing transceiver design for over-the-air function computation," IEEE Wireless Commun. Lett.,, vol. 7, no. 6, pp. 942-945, Dec. 2018.

[7] J. Dong, Y. Shi, and Z. Ding, "Blind over-the-air computation and data fusion via provable wirtinger flow," CoRR, vol. abs/1811.04644, 2018 [Online]. Available: http://arxiv.org/abs/1811.04644

[8] L. Chen, N. Zhao, Y. Chen, F. R. Yu, and G. Wei, "Over-the-air computation for IoT networks: Computing multiple functions with antenna arrays," IEEE Internet Things J., vol. 5, no. 6, pp. 5296-5306, Dec. 2018.

[9] C. Liaskos, S. Nie, A. Tsioliaridou, A. Pitsillides, S. Ioannidis, and I. Akyildiz, "A new wireless communication paradigm through softwarecontrolled metasurfaces," IEEE Commun. Mag., vol. 56, no. 9, pp. 162169, Sept. 2018

[10] S. Hu, F. Rusek, and O. Edfors, "Beyond massive MIMO: The potential of data transmission with large intelligent surfaces," IEEE Trans. Signal Process., vol. 66, no. 10, pp. 2746-2758, May 2018.

[11] M. Di Renzo, M. Debbah, D.-T. Phan-Huy, A. Zappone, M.S. Alouini, C. Yuen, V. Sciancalepore, G. C. Alexandropoulos, J. Hoydis, H. Gacanin et al., "Smart radio environments empowered by ai reconfigurable meta-surfaces: An idea whose time has come," CoRR, vol. abs/1903.08925, 2019. [Online]. Available: https://arxiv.org/abs/1903.08925

[12] Q. Nadeem, A. Kammoun, A. Chaaban, M. Debbah, and M. Alouini, "Large intelligent surface assisted MIMO communications," CoRR, vol. abs/1903.08127, 2019. [Online]. Available: http://arxiv.org/abs/1903. 08127

[13] Q. Wu and R. Zhang, "Intelligent reflecting surface enhanced wireless network: Joint active and passive beamforming design," in Proc. IEEE Global Communications Conf. (GLOBECOM), Abu Dhabi, United Arab Emirates, Dec. 2018, pp. 1-6.

[14] L. Subrt and P. Pechac, "Intelligent walls as autonomous parts of smart indoor environments," IET commun., vol. 6, no. 8, pp. 1004-1010, 2012

[15] E. Basar, "Large intelligent surface-based index modulation: A new beyond MIMO paradigm for 6G," CoRR, vol. abs/1904.06704, 2019. [Online]. Available: https://arxiv.org/abs/1904.06704

[16] W.-K. K. Ma, "Semidefinite relaxation of quadratic optimization problems and applications," IEEE Signal Process. Mag., vol. 1053, no. $5888 / 10,2010$

[17] E. Chen and M. Tao, "ADMM-based fast algorithm for multi-group multicast beamforming in large-scale wireless systems," IEEE Trans. Commun.,, vol. 65, no. 6, pp. 2685-2698, Jun. 2017.

[18] M. Grant and S. Boyd, "CVX: Matlab software for disciplined convex programming, version 2.1," http://cvxr.com/cvx, Mar. 2014.

[19] P. D. Tao and L. T. H. An, "Convex analysis approach to D.C. programming: Theory, algorithms and applications," Acta Math. Vietnamica, vol. 22 , no. 1 , pp. 289-355, 1997. 